Cahiers d'études japonaises

15 | 2008

Guerre, colonialisme et commémoration

\title{
Langue, lecture et école au Japon, sous la direction de C. Galan et de J. Fijalkow
}

\section{Pascal Griolet}

\section{(2) OpenEdition \\ 9 Journals}

\section{Édition électronique}

URL : https://journals.openedition.org/cipango/427

DOI : $10.4000 /$ cipango.427

ISSN : 2260-7706

Éditeur

INALCO

\section{Édition imprimée}

Date de publication : 1 janvier 2008

ISBN : 978-2-85831-177-4

ISSN : 1164-5857

Référence électronique Pascal Griolet, " LANgUE, LECTURE ET ÉCOLE AU JAPON, SOUS LA DiRection DE C. GALAN ET DE J.

FIJALKOW », Cipango [En ligne], 15 | 2008, mis en ligne le 13 novembre 2011, consulté le 30 juin 2021. URL : http://journals.openedition.org/cipango/427 ; DOI : https://doi.org/10.4000/cipango.427

Ce document a été généré automatiquement le 30 juin 2021.

\section{(c) (1) (9)}

Cipango est mis à disposition selon les termes de la Licence Creative Commons Attribution - Pas d'Utilisation Commerciale 4.0 International. 


\title{
Langue, lecture et école au Japon, sous la direction de C. Galan et de J. Fijalkow
}

\author{
Pascal Griolet
}

\section{RÉFÉRENCE}

Langue, lecture et école au Japon, sous la direction de C. Galan et de J. Fijalkow, Ed.

Philippe Piquier, 2006, 405 p. ISBN 2-87730-865-0

1 Cet ouvrage de plus de quatre cents pages présente les Actes d'un colloque international sur l'enseignement de la langue et de la lecture dans la scolarité japonaise, qui s'est tenu en mai 2003 à l'université Toulouse-le Mirail. Il comporte quatre grands volets: les caractéristiques de l'écriture japonaise, les objectifs du système scolaire japonais, l'apprentissage de la lecture, et enfin une dernière partie consacrée à l'histoire et aux pratiques de la lecture.

2 L'avant-propos de Christian Galan et Jacques Fijalkow souligne que la crise de la lecture que l'on constate en France existe aussi au Japon, même si c'est sous des formes atténuées ou différentes, liées à la société, au système scolaire ainsi qu'aux spécificités des deux systèmes d'écriture. C'est pourquoi les questions que soulève l'enseignement de la lecture au Japon sont abordées ici sous différents angles en mettant plusieurs disciplines à contribution (psychologie, linguistique, pédagogie, sociologie, histoire).

Pour ouvrir le premier volet consacré à la langue et à son système d'écriture, JeanPierre Jaffré démontre de façon remarquable la similitude entre les systèmes graphiques $\mathrm{du}$ français et $\mathrm{du}$ japonais en utilisant le concept de «mixité » orthographique. Même si la «surnorme» que constitue l'orthographe par rapport à l'écriture est fixe dans une écriture comme celle du français, l'auteur pose qu'il en existe une aussi dans le cas du japonais, malgré la fluidité théorique qu'on lui reconnaît. Les orthographes du japonais et du français illustrent chacune à leur 
manière le concept de mixité, que deux écritures distinctes coexistent, comme en japonais, ou que les lettres de l'alphabet remplissent tout à tour des fonctions différentes. L'orthographe d'une langue s'appuie sur la "phonographie " d'un côté (c'est en particulier le rôle des kana en japonais) et sur la « sémiographie » de l'autre (c'est là que s'exerce en particulier la fonction des caractères chinois). L'orthographe $\mathrm{du}$ japonais a plutôt tendance à utiliser une écriture opaque sur le plan phonographique pour représenter les signes du lexique tandis qu'elle est transparente dans le domaine grammatical, où l'on trouve les unités linguistiques les plus abstraites. L'orthographe du français associe morphonographie et morphographie alphabétiques, tant du côté du lexique que de la grammaire, ce qui rend les deux domaines, mais spécialement le second, particulièrement difficiles à apprendre et à maîtriser. Si les kana sont des "phonogrammes à fonction syllabique " presque parfaits (à chaque unité phonique correspond une unité graphique et une seule), les caractères chinois ne sont certes pas dépourvus de valeur phonique mais celle-ci n'est pas fiable. Ainsi, si la phonographie du français est plus irrégulière que celle des kana, elle l'est beaucoup plus que celle des caractères chinois. Outre ces deux dimensions, l'auteur examine les aspects psycholinguistiques qui interviennent dans l'acquisition des deux orthographes à partir du modèle dit à double voie : l'une mettant en relation les unités phoniques et graphiques remplit une fonction pré-lexicale tandis que l'autre, post-lexicale, déduit le statut phonographique d'informations orthographiques et sémantiques préalablement traitées.

4 Anne-Marie Christin présente ensuite elle aussi les similitudes incontestables entre les écritures japonaise et européenne. Elles sont d'abord l'une et l'autre des écritures " secondes ", c'est-à-dire empruntées à un système antérieur : l'écriture chinoise pour le japonais, le système consonantique phénicien pour l'alphabet grec, ancêtre de l'alphabet latin. Elles se caractérisent également toutes deux par l'importance qu'elles réservent au phonétisme dans leur structure et, ce qui est plus rare, par la manière dont elles intègrent l'image à certaines de leurs créations.

5 Irène Tamba explique - et de la façon la plus exhaustive possible - à partir de la façon d'écrire un simple verbe, yomu «lire », comment fonctionne un sinogramme utilisé dans l'écriture japonaise et quelles ramifications il y établit. Elle parvient ainsi à aborder la totalité des questions que soulève l'emploi des caractères chinois pour noter la langue japonaise. Irène Tamba conclut de cet examen que l'encodage voire en forçant le trait le « cryptage » de la langue dans la fixité de l'écrit est d'une complexité ou d'une sophistication extraordinaire pour les Occidentaux, mais aussi que le décodage ou « décryptage » est une opération encore plus inconcevable.

6 Patrick Beillevaire présente la langue et les écritures de l'ancien royaume des Ryūkyū, archipel qui constitue aujourd'hui le département d'okinawa. Il brosse un tableau de la spécificité de cet ancien royaume des Ryūkyū, qui fut longtemps un intermédiaire entre la Chine et le Japon, et n'entra que tardivement dans l'univers de l'écrit. Les différents dialectes de cet archipel forment une famille linguistique proche du japonais, sans qu'il y ait cependant possibilité d'intercompréhension. À l'époque du royaume, l'apprentissage de l'écriture était réservé à l'aristocratie et aux fonctionnaires. La langue écrite était en premier le chinois qui était alors linga franca de la région, mais l'écriture japonaise pénétra aussi dans l'archipel et servit pour la communication avec le Japon ainsi pour certains documents, mais seul un petit nombre de lettrés avaient la capacité de converser en chinois ou en japonais. La création du département d'Okinawa 
fut suivie par la mise en œuvre d'une politique intensive de japonisation culturelle et linguistique que l'ensemble de la population allait progressivement faire sienne.

7 Yazawa Makoto, après avoir présenté les traits caractéristiques de l'écriture japonaise, retrace l'historique de la limitation des caractères chinois à l'époque moderne et souligne le rôle que joua la presse écrite dans l'élaboration des normes qui ont cours aujourd'hui en informatique. Il présente la révolution que constitua l'apparition des appareils de traitement de texte au Japon et les différents modes de saisies qui furent élaborés. Il examine enfin la situation présente de la pratique de l'écrit et de son apprentissage à l'heure du téléphone portable et les défis que posent à l'enseignement les nouvelles technologies.

8 Pour les questions liées à l'institution scolaire et à l'enseignement de la lecture, Horio Teruhisa énumère un certain nombre de facteurs qui compliquent la tâche des enseignants. Les directives tatillonnes du ministère de l'éducation et le poids grandissant des comités d'éducation locaux qui contrôlent les manuels scolaires démotivent un certain nombre d'enseignants. Les écoles privées reconnues par le ministère de l'éducation jouissent d'une plus grande autonomie que les écoles publiques. L'école élémentaire doit gérer des enfants de six ans ayant des niveaux de connaissances différents. Les exigences du ministère de l'Education sont en contradiction avec la réduction des horaires et l'allègement des contenus d'enseignements. Les pratiques ludiques et l'imagination devraient jouer un rôle important dans l'apprentissage de la lecture et de l'écriture japonaise. Pour toutes ces raisons, Horio Teruhisa considère qu'il est nécessaire de permettre aux enseignants de mettre en place des pratiques pédagogiques créatrices.

Nanba Hirotaka analyse la compétence vers laquelle doit tendre l'enseignement de la lecture en présentant les résultats d'une enquête internationale menée dans le cadre de l'OCDE ainsi que ceux d'une enquête menée par le ministère de l'Education japonais. Il observe chez les élèves japonais une faiblesse dans l'interprétation lorsqu'elle porte sur un texte dans sa globalité, en particulier lorsqu'il s'agit d'un texte de type " explicatif et argumentatif». Il préconise de réviser la notion de compréhension de l'écrit et de prendre en compte le champ discursif dans lequel le texte s'insère (ce que l'auteur appelle l'espace argumentatif) pour développer une compréhension élargie des textes écrits, qui ne soit pas limitée à l'espace linguistique ou à l'espace discursif.

Claude Lévi Alvarès examine les méthodes d'enseignement dans un collège japonais et la place qui y sont accordées aux différentes «disciplines». Il décrit en détail la formation initiale, les modalités d'embauche et de titularisation, les stages ultérieurs les conditions de travail, et les valeurs partagées par la plupart des membres du corps enseignants en poste dans les collèges publics. La place limitée accordée à la discipline ou la matière enseignée est considérée comme facteur structurant dans la construction des identités professionnelles. Dans les premières années de la carrière d'un enseignant, l'établissement d'une bonne relation avec les élèves constitue une préoccupation centrale. L'immersion dans les techniques de gestion de la classe et la participation aux activités de club sont hautement valorisés. La transmission des savoirs est envisagée comme un aspect de la vie scolaire parmi d'autres. C'est, conclut Claude Lévi Alvarès, la raison pour laquelle de nombreux enseignants n'accordent guère d'importance à une réflexion spécifique sur les méthodes et les techniques d'enseignement. 
11 Shutō Hisayoshi brosse un historique des méthodes d'initiation à la lecture et à l'écriture qui furent élaborées depuis les premiers manuels de lecture de type moderne qui s'inspiraient des manuels scolaires américains dans les années 1870. L'auteur présente chacune des innovations ou expérimentations qui vont suivre pour mieux adapter à l'enseignement de la lecture et de l'écriture les différents manuels: la fixation de l'ordre des signes, la fixation de la série de kana de référence, les réformes orthographiques et les réformes introduites depuis la dernière guerre sous la pression des Américains jusqu'à la situation actuelle.

12 Le volet pédagogie commence par un article de Norimatsu Hiroko qui étudie les pratiques ludiques utilisées pour enseigner les kana chez les enfants âgés de 4 à 6 ans, et en particulier le processus mettant en relation le jeu de mots appelé shiritori (qui correspond au jeu français des queues-de-mots («j'en ai marre, marabout, bout de ficelle...»), le développement de la conscience phonologique et l'apprentissage des kana. La pratique du shiritori nécessite une certaine capacité de composition des sons, et l'extraction du dernier composant phonologique des mots varie selon le niveau d'apprentissage de la lecture. Les différentes études menées sur la question permettent de penser qu'il pourrait y avoir des relations de causalité entre les facteurs permettant le jeu de shiritori, le développement de la conscience phonologique et le niveau de l'acquisition de la lecture.

13 Kawakami Sachiko examine les débuts de l'apprentissage des caractères chinois à l'école élémentaire. Ce n'est qu'à partir de la fin du deuxième semestre que les élèves de la première année de l'école élémentaire (équivalent du cours préparatoire français) commencent à apprendre les caractères chinois. Ils en apprendront 72 durant cette première année. La méthode d'enseignement qui a cours à l'école élémentaire consiste, notamment durant cette période de l'entrée dans l'écrit, à enseigner l'origine des caractères afin de mieux faire saisir leur sens aux enfants. Connaître pour chaque signe les différentes étapes de son évolution graphique permet aux enfants de mieux prendre conscience que la signification des formes abstraite des sinogrammes. Les pratiques pédagogiques mises au point dans ce but permettent aux enfants d'apprendre les caractères chinois en se les appropriant.

Tsukada Yasuhiko présente l'évolution des théories sur l'enseignement de la lecture au Japon au travers des manuels scolaires de l'école élémentaire. Il décompose pour cela le domaine de la lecture en quatre niveaux : 1 . reconnaissance et acquisition du système d'écriture (niveau de la phonétique) 2. reconnaissance des mots (niveau de la perception des mots et de la compréhension de leur sens) 3.compréhension des phrases (niveau de la compréhension syntaxique et sémantique de la phrase) 4. compréhension du texte (niveau de la compréhension de la structure du sens du texte). Tsukada Yasuhiko examine en particulier les manuels des niveaux 1 et 2 afin de mettre en évidence le grand écart qui les sépare du niveau 3. On considère en effet au Japon que l'apprentissage des niveaux 1 et 2 commence avant l'entrée à l'école primaire et l'encadrement pédagogique des cours se concentre très tôt sur le niveau 3 , la compréhension des phrases. En outre, la frontière entre les niveaux 3 et 4 n'est pas clairement définie, ce qui soulève diverses questions qu'il faudra résoudre.

Pour le volet histoire et pratiques et de la lecture, Kobayashi Akemi décrit en détail les pratiques liées à lecture dans le Japon du XVI ${ }^{\mathrm{e}}$ et $\mathrm{du} \mathrm{IX}^{\mathrm{e}}$ siècles en commençant par les procédés d'analyse phonétique des sinogrammes avant que fut mis au point le syllabaire japonais. Elle examine ensuite les difficultés rencontrées à ces époques pour 
prononcer de la même manière ces signes venus du chinois à différentes époques. La politique qui fut menée pour mettre un terme à l'anarchie et unifier les usages en se conformant au modèle du chinois de l'époque, la langue des Tang, est à l'origine de la situation présente où coexistent plusieurs prononciations sino-japonaises pour un même sinogramme.

Peter Kornicki présente l'essor de l'édition au XVII ${ }^{e}$ siècle et en particulier l'édition d'ouvrages destinés à un public féminin. L'imprimerie, qui est connue au Japon depuis le viII ${ }^{\mathrm{e}}$ siècle, ne se développe en activité commerciale qu'à partir du XVII ${ }^{\mathrm{e}}$ siècle. Les livres deviennent alors des objets familiers facilement accessibles, tout particulièrement dans les villes. A partir des années 1650 apparaît un nouveau marché constitué de livres destinés aux femmes. Au même moment, les lettrés commencent à s'inquiéter au sujet du contenu de ces ouvrages. A leur yeux on ne s'y intéresse qu'aux relations sentimentales et les règles éthiques que doivent suivre, à leur yeux, les femmes, y sont ignorées sinon enfreintes. Peter Kornicki observe aussi les nombreuses illustrations où sont représentées des femmes en train de lire et observe enfin l'importance que l'on accordait à la lecture chez les courtisanes de haut rang. Il constate enfin que l'affirmation des goûts japonais que l'on trouve dans les publications destinées aux femmes est contemporaine d'une éducation des hommes qui était, elle, fortement influencée par la pensée chinoise.

Richard Rubinger se penche sur l'illettrisme dans le Japon de Meiji tout d'abord à partir des rapports annuels du ministère de l'éducation qui sont établis à partir de 1873 et qui à partir de 1877 mentionnent pour trois régions du pays la capacité d'écrire son propre nom dans la population. Les tableaux qu'en tire Richard Rubinger montre que le taux d'illettrisme du début de Meiji va en diminuant. Il établit aussi des corrélations entre hommes et femmes sur ce plan. Il se penche ensuite sur les rapports annuels statistiques du ministère de l'armée à partir de 1889. Après avoir présenté les tests auxquels sont soumis les conscrits, il dresse des tableaux qui montrent de grands écarts selon les régions, voire les quartiers dans le cas des villes. Il constate à nouveau une diminution sensible des illettrés au fil du temps avec des variations qu'illustrent tableaux et cartes.

Christian Galan analyse les discours tenus au Japon sur le faible niveau d'illettrisme de ce pays par rapport à tous les autres et qui laissent entendre que tous les Japonais savent lire et écrire. Il cherche les origines de ce genre de discours dans la confusion régnant entre le taux élevé de la scolarisation à l'époque d'Edo et le niveau de maitrise de l'écrit. Cette confusion en est venu à constituer un stéréotype qui fut repris ensuite par les étrangers parlant du Japon. Christian Galan présente ici les différentes enquêtes qui été menées à l'époque moderne pour tenter de mesurer la capacité de lire et d'écrire au Japon. Il montre la difficulté de mesurer cette capacité en raison de la nature de l'écriture qui a plusieurs niveaux depuis l'usage des signes syllabiques jusqu'à celui de milliers de sinogrammes. Christian Galan reproche au ministère de l'éducation japonais de ne pas engager d'enquête scientifique sur le sujet et voit dans ce mythe de l'absence d'illettrisme au Japon un discours d'exclusion d'une part et de nationalisme de l'autre.

Jacques Fijalkow conclut l'ensemble par une postface en dégageant des pistes de recherches sur l'écriture, la lecture et l'enseignement et en appelant à des recherches communes sur ces points. 